\title{
9
}

\section{Integrating Sewer Inspection Data into SWMM Model Calibration}

\author{
Fang Cheng, Gregory R. Barden, Edward H. Burgess and \\ Julie A. McGill
}

Developing and calibrating a large scale SWMM model in coordination with sewer cleaning and CCTV inspection can be a challenging, labor-intensive and time-consuming task. The common model calibration approach on closed conduit hydraulics involves estimating roughness coefficients and sediment depths throughout the sewer system, when detailed sewer condition parameters are available from CCTV inspections. If those conditions are changing throughout the calibration period due to sewer cleaning, this task becomes more complicated.

For the City of Columbus's Livingston/James Sewer System I/I Remediation Project, the project team developed a comprehensive database application tool to integrate the condition parameters from 130 miles of sewer inspection into the model calibration process. The project's SWMM EXTRAN model contains 2,900-conduits ranging from 8 in. (203 $\mathrm{mm})$ to 102 in. $(2590 \mathrm{~mm})$ in diameter. This tool takes defects recorded for each of the sewer segments being inspected, and relates them to appropriate Manning's roughness coefficients and/or sediment depths used in the calibration process. For sewers with multiple defects, the defects are rated and the calibration is based on the most severe defects. The time that pipe cleaning was performed is also taken into account because three calibration

Cheng, F., G. Barden, E. Burgess and J.A. McGill. 2008. "Integrating Sewer Inspection Data into SWMM Model Calibration." Journal of Water Management Modeling R228-09. doi: 10.14796/JWMM.R228-09. (C) CHI 2008 www.chijournal.org ISSN: 2292-6062 (Formerly in Reliable Modeling of Urban Water Systems. ISBN: 978-0-9808853-0-9) 
storms were selected at different times during the cleaning process. This new tool helps to shorten the calibration time and still maintains accuracy, while accounting for approximately 43,000 sewer defects, including roots, in the SWMM model calibration.

\subsection{Introduction}

\subsubsection{Livingston/James Sewer System I/I Remediation Project}

The Livingston/James Sewer System Inflow and Infiltration Remediation Project is the first of the ten Inflow/Infiltration projects designed to address wet weather problems in the City of Columbus. The general goal of the project is to clean and CCTV (Closed Circuit Televise) all sewers in the project area, to investigate the sources of inflow and infiltration from both private and public sides, and to identify locations and causes of sewage overflowing from manholes, sanitary reliefs, sewerage system surcharging, and backup into basements. The final objective is to recommend costeffective improvements to the sanitary collection system to mitigate and/or eliminate these occurrences for selected design criteria.

An important element of the project is developing a computer model of the existing sanitary sewer system within the Livingston/James project area and applying the model to study and evaluate the hydraulic performance of the sewer system and to explore rehabilitation alternatives. Using PCSWMM 2006, the model was built comprising 2,897 conduits, including all pipes eight in. $(203 \mathrm{~mm})$ or larger in diameter, 2,865 junctions, and 2082 catchments in 27 flow meter basins. This chapter focuses on the process of model calibration, specifically how to integrate sewer inspection data into the calibration process to improve reliability and cost-effectiveness. The effects of sewer cleaning are also discussed.

\subsubsection{Sewer Inspection Process}

As part of this project, over 3,000 sanitary sewer pipelines, 8 in. diameter and larger (a total of 720,000 feet/219,512 $\mathrm{m}$ in length), were cleaned and inspected over a nine month period. Field crews recorded conditions of the sewer during the sewer inspection process using a standardized Pipeline Assessment and Certification Program (PACP) code. PACP codes were developed by the National Association for Sewer Service Companies, and 
the purpose is to provide a standardized and consistent way to evaluate sewers. One of the computer programs used to record the inspection is PipeTech. A complete database including all the inspections was created in PipeTech and converted into MS Access format.

\subsubsection{Challenges of Model Calibration}

One of the primary challenges of the model calibration was the overlap of the flow monitoring for calibration and the sewer cleaning and inspection period. Because of schedule constraints, the flow monitoring program could not be started sufficiently ahead of the cleaning, nor after the cleaning was over. The three selected calibration storms occurred during three different stages of cleaning: $25 \%, 60 \%$ and $95 \%$ complete.

The challenge was then to estimate the changing variables in the model. For closed conduit hydraulic calibration, Manning's roughness coefficient and sediment depth for each conduit need to be estimated. The EXTRAN model consists of 2,897 conduits; therefore approximately 6,000 variables need to be estimated. Typically, the sewer condition is unknown, however we had the advantage of better understanding the sediment depth and sewer conditions throughout the entire area from the sewer inspections. Therefore, it was possible to estimate sediment depth.

\subsection{Methodology}

Pipe inspection can provide valuable information about the pipe conditions for model calibration. In addition to providing information on sewer conditions, CCTV inspection can provide a close-up view of important structures such as diversion structures and manholes with multiple inflow and /or outflow pipes.

To estimate sediment depth and Manning's $\mathrm{n}$ for calibration, the project team began by categorizing pipes based on inspection data. A database, including all the pipe inspections, was created using MS Access. All the pipes were then divided into seven categories based on the PACP codes. Because some pipes have multiple defects, the categories were given a priority rank. Following the flowchart presented in Figure 9.1, cleaned pipes were first separated from the non-cleaned pipes. Then, the non-cleaned pipes were further categorized into excessive heavy cleaning (EHC) for heavy deposits, roots ball, medium roots, deposit settled, obstacles, and deposit 
attached to pipes. Finally, Manning's n and/or sediment depth were estimated for each category of pipes instead of each individual pipe.

\subsubsection{Estimating Sediment Depth}

One approach attempted for estimating sediment depth was from the CCTV videos. For some pipes, the videos show an obvious water mark indicating the water level during dry weather flow before cleaning. However, it would have taken at least 1,200 hours to review all the videos. The other concern is that sometimes the videos were recorded during cleaning. In this case, the water level shown in the video is not the water level during dry weather flow, because the jet nozzle spraying high pressure water into the pipe causes the water level to rise.

The other source of sediment depth data is the daily logs and comments of each inspection. The survey crews estimated sediment depth and recorded that information as comments wherever a significant deposition was found. This data source proved more accurate and efficient, and was used for sediment depth estimation.

\subsubsection{Estimating Manning's $n$}

Based on engineering experience, Manning's $n$ of 0.013 was applied to clean sanitary sewer pipes with no defects. For the dirty pipes and pipes with defects, Manning's n values were iterated during the calibration process. A range of Manning's $\mathrm{n}$ values were given to pipes with heavy deposits, roots ball, medium roots, and obstacles based on the percentage of blockage. A trial-and-error calibration process was performed until the computed flow and water level matched the observed data at the flow monitoring sites.

\subsubsection{Using CCTV to Understand System Hydraulics}

CCTV videos can provide detailed information on system hydraulics especially at complicated structures and locations. For instance, model simulation at manholes with multiple outflow conduits is challenging due to a lack of information on why flows were split in a certain ratio, although downstream flow monitoring provides flow data in the outflow branches. With CCTV video visualizing the hydraulics at those locations, the modeling personnel can answer the questions of how and in what proportion flows were split. 


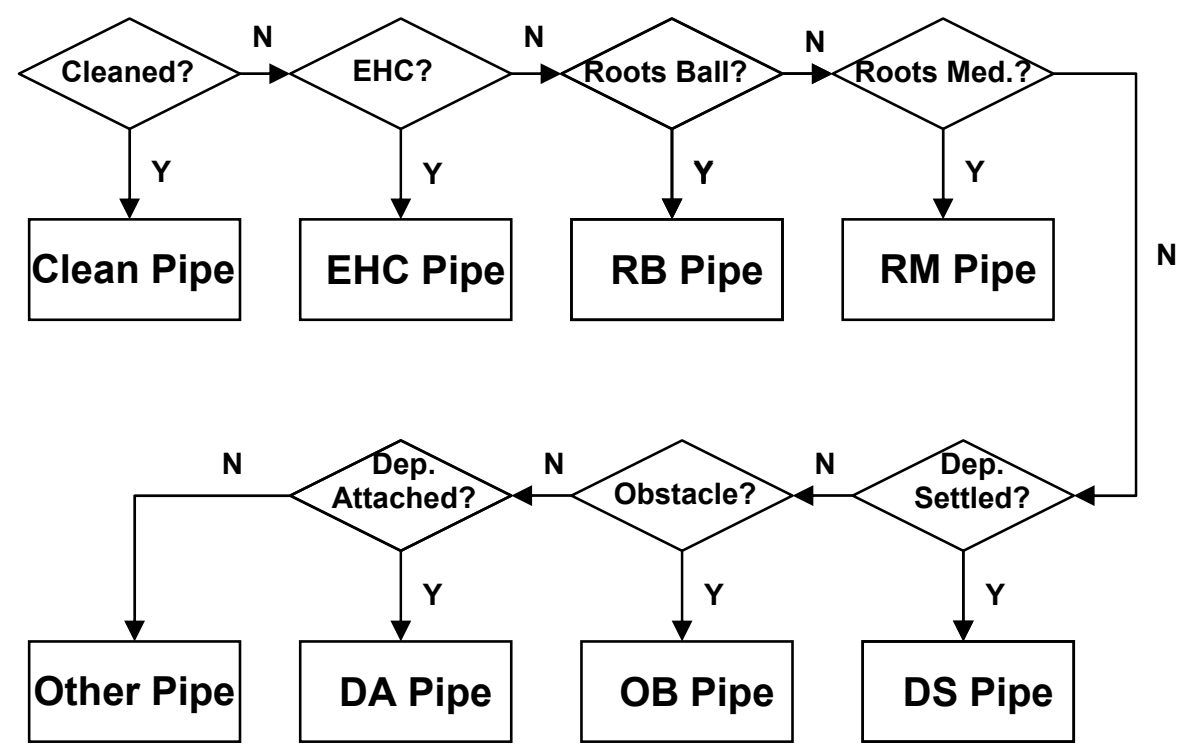

\subsection{Results}

Figure 9.1 Ranking of the pipe defects.

For the sanitary sewer system within the project area, the estimated sediment depths are between 0.6 to 6 in. (15-152 mm), and Manning's n values range from 0.013 to 0.016 , as shown in Table 9.1. Note: for the post-cleaning model, Manning's n was returned to 0.013 and sediment depths set to zero. For pipes with roots ball, medium roots, and obstacles, Manning's coefficients lie within a range. The range is determined from the percentage of blockage recorded during pipeline inspections.

To quantify the effects of sewer cleaning, the full pipe carrying capacity was calculated and pre-cleaning and post-cleaning conditions compared by using Manning's equation (Table 9.2). The results show that due to lack of cleaning, full pipe carrying capacity was reduced by $25 \%$ for sewers that are 10 in. $(254 \mathrm{~mm})$ and larger and about $7.9 \%$ for 8 in. $(203 \mathrm{~mm})$ sewers. In one example, the computed results also show that over $50 \%$ of the system surcharged during a moderate storm (March 28, 2005) before cleaning, while only $10 \%$ of the system would still be surcharging after cleaning (Figures 9.2 and 9.3). At some locations within the system, the hydraulic grade line could decrease up to $4 \mathrm{ft}$. after the cleaning (Figure 9.4). 
Table 9.1 Ranges of Sediment Depth and Manning's n.

\begin{tabular}{lll}
\hline Pipe Categories & Sediment Depth (inch) & Manning's n \\
\hline Clean & 0 & 0.013 \\
\hline Excessive heavy cleaning & $5-6$ & $0.015-0.016$ \\
\hline Roots ball & 0 & $0.014-0.016$ \\
\hline Roots medium & 0 & $0.0135-0.0155$ \\
\hline Deposits settled & $0.6-2$ & 0.015 \\
\hline Obstacles & 0 & $0.0132-0.015$ \\
\hline Deposit attached & 0 & 0.0135 \\
\hline Others & 0 & 0.015 \\
\hline
\end{tabular}

Table 9.2 Full pipe carrying capacity loss caused by lack of cleaning.

\begin{tabular}{ll}
\hline Pipe Diameter (inch) & Average \% Capacity Loss \\
\hline 8 & 7.9 \\
\hline 10 & 31.8 \\
\hline 12 & 29.7 \\
\hline 15 & 27.3 \\
\hline 18 & 25.4 \\
\hline 21 & 29.7 \\
\hline 24 & 28.3 \\
\hline 30 & 24.1 \\
\hline 36 & 27.2 \\
\hline 42 & 25.0 \\
\hline 48 & 18.5 \\
\hline 54 & 25.5 \\
\hline 66 & 22.6 \\
\hline 96 & 19.3 \\
\hline
\end{tabular}

\subsection{Conclusions and Discussions}

Pipe inspection can provide valuable information about the pipe conditions for model calibration. However, consistency between different surveyors is the key to keeping the inspection database valuable. The efficiency of data analysis and model calibration is highly dependent on the consistent use of the PACP condition code and pipe naming system recorded during inspection. 


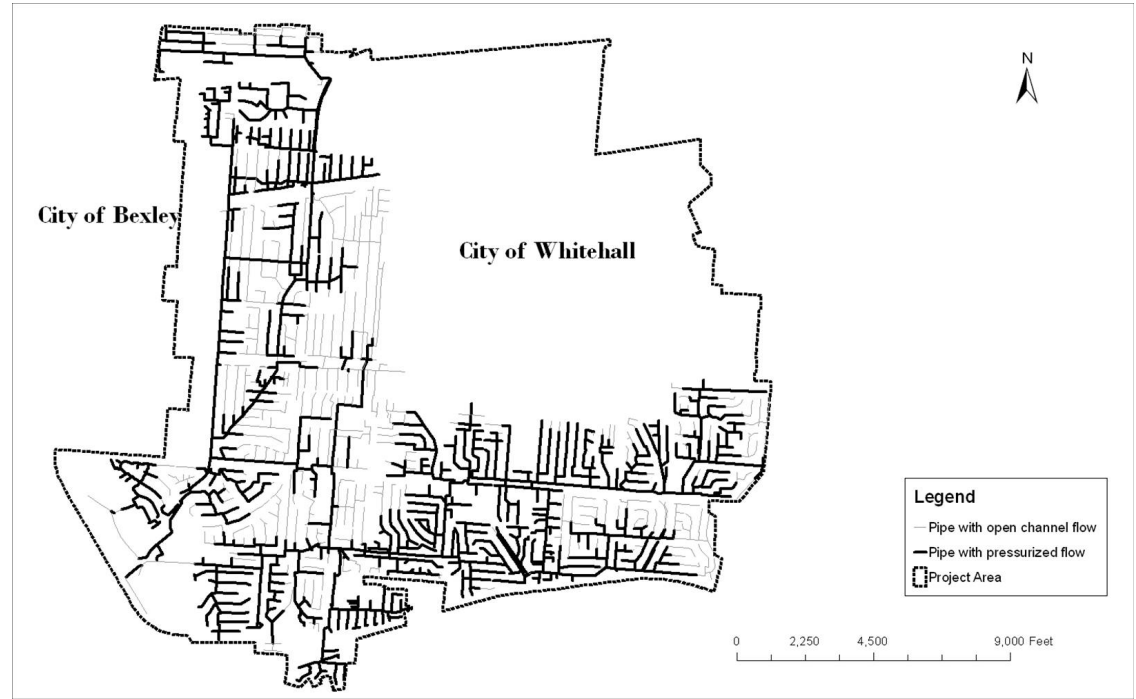

Figure 9.2 Locations of pipes with pressurized flow, pre-cleaning

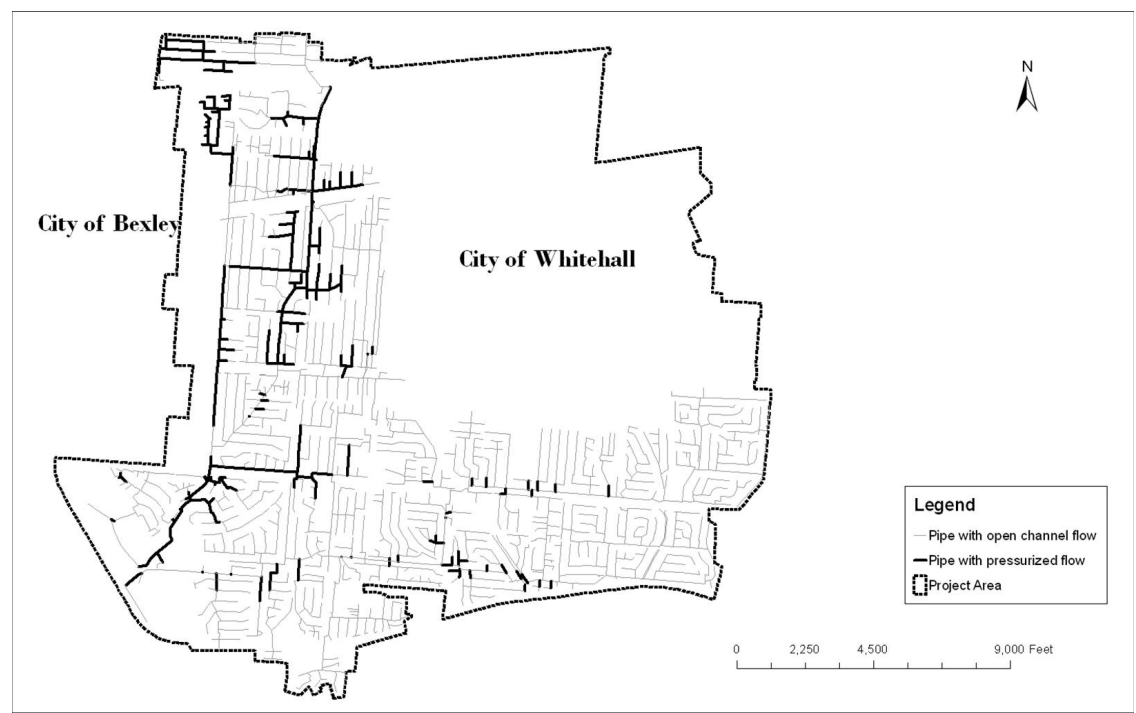

Figure 9.3 Locations of pipes with pressurized flow, post-cleaning 

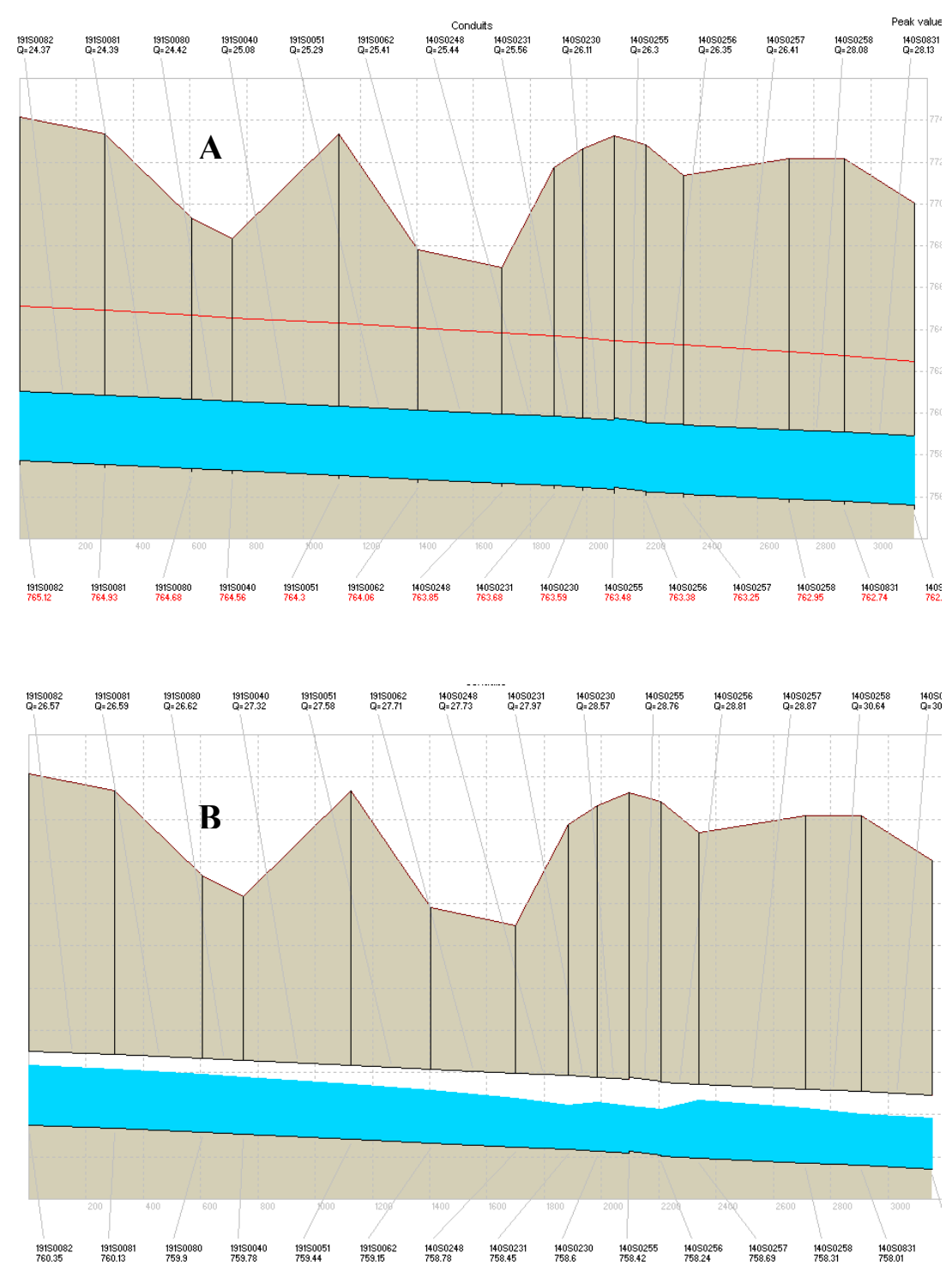

Figure 9.4 Change of hydraulic grade line, A, pre-cleaning and B, post-cleaning 
For sediment depth estimation, surveyors should estimate sediment depths at the site before the cleaning, especially if significant deposits are discovered. This approach provides more accurate information on sediment depths, one of the key variables for model calibration, than can be obtained from the inspection videos themselves.

It is also suggested that the surveyors carefully inspect diversion structures and manholes with multiple inflow and/or outflow pipes. It was found that the flow split ratio at those structures and manholes significantly impacts the hydraulics calibration. An onsite video can provide detailed information on how flow is split at those structures and manholes, which will give the engineers a better understanding of the hydraulics at those locations and eventually facilitate model calibration.

\section{Acknowledgments}

The authors would like to acknowledge CDM and the City of Columbus, Ohio for their contributions to this project and John Schroeder for sharing his knowledge and experience on CCTV inspections.

\section{References}

City of Columbus, Ohio. (2005). Wet Weather Management Plan 
